\title{
Chemistry of the Solar System Revealed in the Interiors of the Giant Planets
}

\author{
Jonathan I. Lunine ${ }^{1}$ \\ ${ }^{1}$ Department of Astronomy, Cornell University, \\ 402 Space Sciences Building, Ithaca, NY 14853 USA \\ email: jlunine@astro.cornell.edu
}

\begin{abstract}
The giant planets of our solar system contain a record of elemental and isotopic ratios of keen interest for what they tell us about the origin of the planets and in particular the volatile compositions of the solid phases. In situ measurements of the Jovian atmosphere performed by the Galileo Probe during its descent in 1995 demonstrate the unique value of such a record, but limited currently by the unknown abundance of oxygen in the interior of Jupiter-a gap planned to be filled by the Juno mission set to arrive at Jupiter in July of 2016 . Our lack of knowledge of the oxygen abundance allows for a number of models for the Jovian interior with a range of $\mathrm{C} / \mathrm{O}$ ratios. The implications for the origin of terrestrial water are briefly discussed. The complementary data sets for Saturn may be obtained by a series of very close, nearly polar orbits, at the end of the Cassini-Huygens mission in 2016-2017, and the proposed Saturn Probe. This set can only obtain what we have for Jupiter if the Saturn Probe mission carries a microwave radiometer.
\end{abstract}

Keywords. giant planets, noble gases, water, oxygen

\section{Introduction}

The giant planets of our solar system were traditionally considered to be cosmic oddballs, interesting specimens of the solar nebula (protoplanetary disk) from which the Sun and planets formed some 4.55 billion years ago. Indeed, speculation based on pioneering models of planetary accretion suggested that giant planet formation might be relatively rare Wetherill (1992). However, the current tally of discoveries of giant planets indicates that roughy $10 \%$ of Sun-like stars possess planets within an order of magnitude the mass of Jupiter Marcy and X (2010). Ground-based microlensing survey have found hundreds of unbound (free-floating) or loosely bound planets with masses of order that of Jupiter; given the efficiency of detection, this implied that two giant planets have formed for every star in the galaxy OGLE and M (2011).

Since giant planets appear to be a common product of star formation, albeit with only a minority remaining in tightly bound orbits around their parent stars, understanding the composition and structure of such bodies should be an important goal of astrophysics. Study of Jupiter, Saturn, Uranus and Neptune no longer can be regarded as a planetological specialty, but rather as an important branch of astrophysics that informs the study of the countless numbers of giant planets likely to be present in the cosmos.

Of particular interest in this regard are in situ measurements of composition, by entry probe, combined with remote sensing of deep atmospheric species and mapping of gravitational and magnetic fields-remote sensing that requires close range (though see dePater et al. (2001) for examples of microwave radiometry of the giant planets done from Earth). The next decade promises an unprecedented opportunity to build on the Galileo entry probe mission of 1995 (Fig. 1), through close orbital sensing of Jupiter and Saturn from Juno and Cassini. The possibility of a Saturn entry probe akin to the Galileo 
Jupiter probe, in the decade following, would complete the basic set of measurements needed to characterize in detail both of the solar system's largest planets.

Here I will focus on the chemical and isotopic information contained in Jupiter and Saturn that informs the details of solar system formation, and the observations that will deliver this information in the near future.

\section{An overview of the chemical and isotopic signatures of Jupiter and Saturn}

A summary of the key chemical and isotopic signatures in Jupiter and Saturn is given in Table 1. Jupiter's composition was measured in situ down to 22 bars; Saturn's was measured remotely by a variety of ground- and space-based instruments.

$\underline{\text { Helium. }}$. The helium abundance is thought to have been uniform throughout the protosolar nebula during the formation of the planets and, therefore, any variation from the generally accepted protosolar value of 0.28 (expressed as a mass fraction; Profitt 1994) should reflect processes internal to the giant planets themselves. The Jovian value is close to but slightly below the protosolar one, with error bars based on the disagreement between the mass spectrometer and the helium detector (refractometer) instruments. The Saturnian value is well below the solar value but with larger error bars because it is derived from remote sensing data. The values given here are from preliminary analyses of

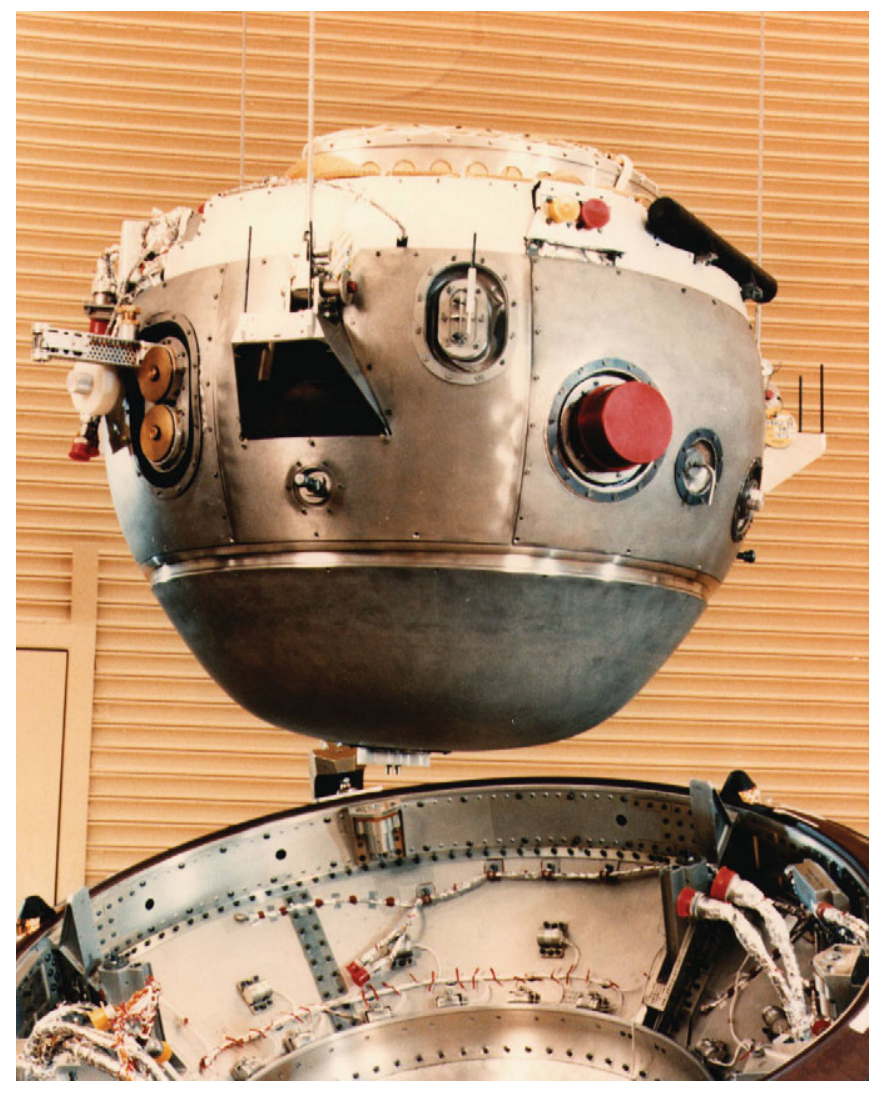

Figure 1. Descent module of the Galileo probe, suspended above the deceleration module/aeroshell. Diameter of the descent module is about 0.9 meters (NASA). 
Table 1. Some chemical and isotopic signatures in Jupiter and Saturn ${ }^{1}$

\begin{tabular}{|c|c|c|c|c|}
\hline $\mid$ Element $\mid$ & $\left|\begin{array}{c}\text { Abundance in } \\
\text { Jupiter }(\text { solar }=1)\end{array}\right|$ & $\begin{array}{l}\text { Isotopes } \\
\text { in Jupiter }\end{array}$ & $\left|\begin{array}{c}\text { Abundance in } \\
\text { Saturn }(\text { solar = 1) }\end{array}\right|$ & $\begin{array}{l}\text { Isotopes } \\
\text { in Saturn }\end{array}$ \\
\hline |hydrogen | & defined $=1$ & $D / H=2.25_{-}^{+} 0.35 \times 10^{-5}$ & defined $=1$ & $\left|D / H=1.70_{-0.45}^{+0.75} \times 10^{-5}\right|$ \\
\hline helium & $0.9_{-}^{+} 0.5$ & $\left|{ }^{3} \mathrm{He} /{ }^{4} \mathrm{He}=1.66_{-}^{+} 0.05 \times 10^{-4}\right|$ & $\approx 0.6-0.86$ & $?$ \\
\hline neon & $0.059_{-}^{+} 0.004$ & $?$ & $?$ & $?$ \\
\hline argon & $3.0_{-}^{+} 1.0$ & $?$ & $?$ & $?$ \\
\hline |krypton | & $2.03_{-}^{+} 0.38$ & $?$ & $?$ & $?$ \\
\hline xenon & $2.11_{-}^{+} 0.40$ & $?$ & $?$ & $?$ \\
\hline carbon & $3.82_{-}^{+} 0.66$ & ${ }^{12} C /{ }^{13} C=92.6_{-}^{+} 0.4$ & $10.4_{-}^{+} 0.4$ & $91.8_{-5.3}^{+5.5}$ \\
\hline nitrogen & $4.90_{-}^{+} 1.87$ & ${ }^{14} N /{ }^{15} N=440+60$ & $4.5_{-}^{+} 1$ & $?$ \\
\hline oxygen & $0.29_{-}^{+} 0.09$ & $?$ & $?$ & $?$ \\
\hline sulfur & $2.88_{-}^{+} 0.69$ & $?$ & $\approx 17$ & $?$ \\
\hline
\end{tabular}

Notes:

1 Jovian abundances from various sources as tabulated and cited in Marty et al. (2008) except for helium which is from von Zahn et al. (1998) and Niemann et al. (1998), argon from Mahaffy et al. (2000), D/H as reported in Fouchet et al. (2009), and oxygen, which is from Wong et al. (2008), using the solar abundances of Grevesse et al. (2005) (but see also Asplund et al. 2009). Solar $\mathrm{He} / \mathrm{H}_{2}$ is from Profitt 1994. Saturnian abundances taken from Fouchet et al. (2009) based on various published and unpublished sources.

Cassini infrared data, which relies on the sensitivity to the helium fraction of the shape of the continuum infrared absorption. However, this analysis does not yield the same result as a different analysis which combines data from radio occultations with infrared data, and further there is disagreement with the same type of analysis from the earlier Voyager flybys. This complex situation is well described in Fouchet et al. (2009).

Despite the uncertainties the interpretation seems straightforward, which is that helium is not completely miscible with hydrogen at the temperatures relevant to the interiors of Jupiter and Saturn, and the zone of immiscibility is larger in Saturn than it is in Jupiter-consistent with the lower temperature at a given pressure in Saturn relative to Jupiter. Immiscibility of helium means that droplets of helium form in Saturn's deep interior; the sinking of these results in enrichment of helium in the deep interior while the outer layers are depleted (Stevenson and Salpeter, 1977)). The sinking of the droplets releases gravitational energy which should be measurable as an added heat source, and indeed the thermal emission from Saturn (Hanel et al. 1983)) is larger than expected for a planet which is the age of the Sun. Jupiter, on the other hand, has an infrared emission consistent with its assumed age of 4.55 billion years, assuming that the emissions sources are only thermalized solar energy and the original virtualized energy of formation of the self-gravitating body. While the coarse outlines of this story seem complete, there remain disagreements on the details (such as whether helium differentiation is occurring in Jupiter as well) because of uncertainties in the hydrogen-helium equation of state at high pressure (Hubbard et al. 2009, Wilson and Militzer 2010 ).

The ratio of the light isotope of helium ${ }^{3} \mathrm{He}$ relative to hydrogen is lower in Jupiter than the generally accepted value for both the solar wind (a sample of gases from the interior of the Sun today) and the local interstellar medium, while the $\mathrm{D} / \mathrm{H}$ value is larger. The sum of the ${ }^{3} \mathrm{He}$ and deuterium abundances, normalized to the hydrogen abundance, is within the (somewhat large) error bars the same as the solar and ISM values; see (Fig. 2) During stellar nucleosynthesis deuterium is destroyed and ${ }^{3}$ He produced. Since 
the Sun's deep interior has been undergoing fusion, and the local interstellar medium is contaminated with material from massive stars that similarly reflects fusion processes within Population 1 stars, a sample of gas unaltered by fusion from the time of the solar system's formation should have lower ${ }^{3} \mathrm{He}$ and higher deuterium than that found in the solar wind (a sample of the solar interior) or local ISM, but the sum should be the same. As figure 2 shows, this is indeed the case for Jupiter. (Owen and Encrenaz 2003). A similar test for Saturn awaits measurement of the ${ }^{3} \mathrm{He}$ abundance, which can only be done in situ by an entry probe.

Noble gases and $C, N, S, P$ bearing species. Besides that of helium, the abundances of all of the other noble gases (neon, argon, krypton, and xenon) have been measured in Jupiter by the Galileo mass spectrometer. Remote sensing measurements of these species in the well-mixed part of the atmosphere is extremely difficult, and hence there are no values reported for Saturn. Initial work analyzing the data used solar abundances that suggested a uniform enrichment of argon, krypton and xenon relative to solar (Mahaffy et al. (2000)), but more recent updates to the solar elemental abundances (Asplund 2009) lead to a slightly larger enrichment of argon relative to solar compared to that of krypton and xenon. The neon abundance $\mathrm{Ne} / \mathrm{H}_{2}$ is strongly depleted relative to solar, and unlike water (see below) this is not a meteorological effect in the observable atmosphere. It is also unlikely to be a depletion in the nebula, since the $\mathrm{He} / \mathrm{H}_{2}$ ratio is not similarly depleted. Most likely it is a miscibility effect in the deep interior (Wilson and Militzer 2010). Unlike neon, which is simply too volatile to incorporate in icy planetesimals, argon, krypton and xenon directly condensed or were trapped or adsorbed in water ice.

There has been a lot of debate on the question of the origin of the heavy noble gas enrichments, as well as that of carbon, nitrogen, sulfur and phosphorous. The uniformity is most easily explained if all of the species condensed out as ices, and given their differing

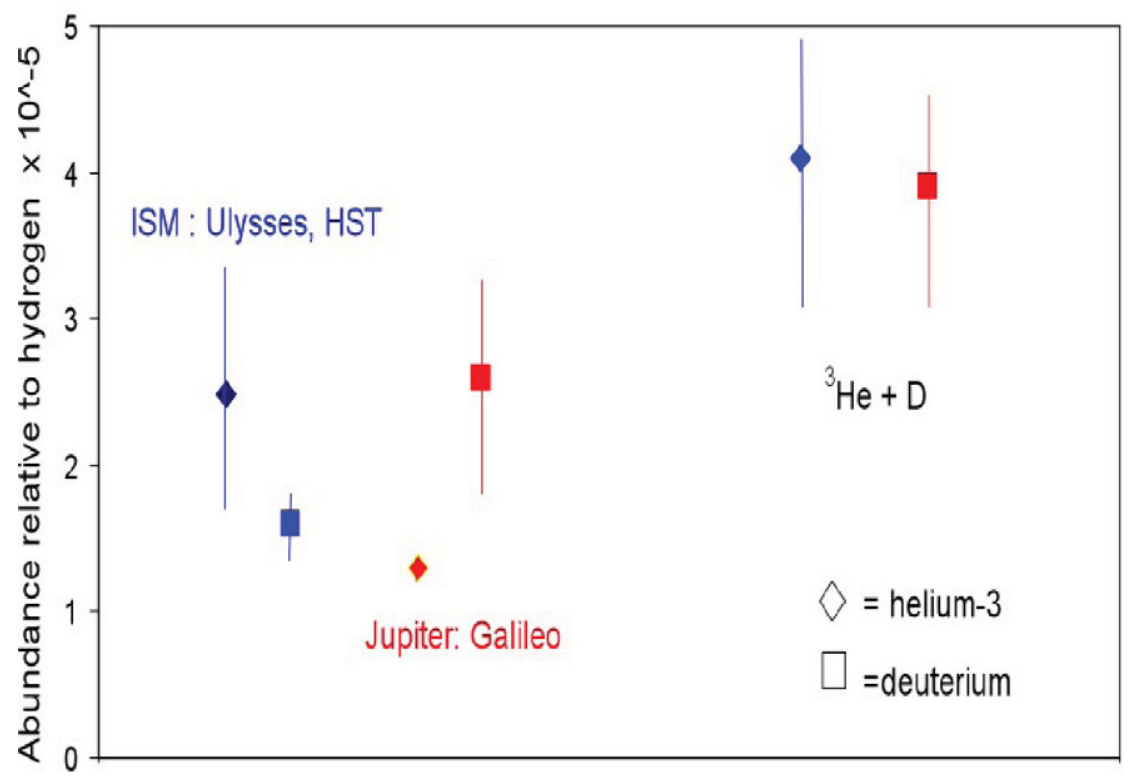

Figure 2. ${ }^{3}$ He and deuterium (D) abundances in Jupiter, and their sum, plotted relative to the hydrogen abundance in Jupiter. The red points and error bars are the Galileo probe data; the blue come from Ulysses solar wind measurements and Hubble Space Telescope observations of the local interstellar medium. Figure based on an argument in Owen and Encrenaz (2003). 
volatilities, this required very low temperatures in the protoplanetary disk, $30 \mathrm{~K}$ or perhaps lower (Owen et al. 1999). An alternative model invoked trapping in clathrate hydrate (Hersant et al. 2004). Revisions to solar abundances led to enrichments that were no longer quite so uniform (Grevesse et al. (2005)), which allowed the clathrate model to more easily fit the data (Mousis et al. 2009). The clathrate model requires nebular temperatures of between $30-40 \mathrm{~K}$, not quite as cold as direct condensation, but also implies a larger fraction of water ice accompanying the carbon-, nitrogen- and sulfurbearing species than does the direct condensation model.

The carbon isotopic ratio ${ }^{12} \mathrm{C} /{ }^{13} \mathrm{C}$ in Jupiter is similar to the terrestrial value in those species in Jupiter not affected by photochemical processes, while the ${ }^{14} \mathrm{~N} /{ }^{15} \mathrm{~N}$ is enriched by a factor of two relative to terrestrial (Owen and Encrenaz, 2003). This suggests that while the carriers of carbon to the Earth and to Jupiter were perhaps similar, those of nitrogen were not.

Oxygen. The deep oxygen abundance in Jupiter is not known because the abundance of water-the primary carrier of oxygen in the Jovian atmosphere-is in principle strongly affected by condensation and rainout associated with meteorological processes (Lunine and Hunten 1987), by advection (Showman and Dowling 2000), or both. The Galileo probe fell into a relative dry region known as a "hot spot" (for the excess brightness observed in such regions at $5 \mu \mathrm{m}$ wavelengths), and the water abundance was observed to increase with depth, or equivalently, with pressure. The sparseness of the measurements made it impossible to know whether the water had "leveled out" at a value corresponding to $1 / 3$ solar or would have increased further had the probe returned data below the 21 bar level where communication ceased. Predictions for either the direct condensation model or the clathrate model are for oxygen abundances several to many times solar in the Jovian deep interior.

\section{A carbon-rich Jupiter}

Although atmospheric explanations for the water abundance in Jupiter are attractive, one must not rule out the possibility that water truly is depleted in the Jovian interiorthat is, the oxygen-to-hydrogen ratio in Jupiter is subsolar. One justification for making such a case is that a planet with a $\mathrm{C} / \mathrm{O}>1-\mathrm{a}$ "carbon-rich planet"-has been discovered (Madhusudhan et al. 2011a), companion to the star WASP12a with a C/O ratio of 0.44 , roughly solar. One explanation is that the portion of this system's protoplanetary disk was somehow depleted in water at the time the planet formed and acquired its heavy element inventory (Madhusudhan et al. 2011). Prior to this discovery, the possibility of a carbonrich Jupiter was considered on the basis of the Galileo results alone by Lodders (2004), who proposed that the point in our protoplanetary disk at which water ice condensedthe snowline- might have been further from the Sun than the point at which Jupiter formed, and volatiles adhering to solid organics rather than water ice were carried into Jupiter. Indeed, for an increasingly oxygen-depleted (or carbon-enriched) protoplanetary disk, the point at which water ice forms from water vapor moves progressively outward (Fig. 3). One difficulty with this hypothesis is the presence of water in the asteroid belt in the form of the main belt comets (Hsieh and Jewitt 2006), although the relatively high rock-to-ice ratio in the Jovian vs Saturnian satellite systems might be an argument in favor.

Mousis et al. (2011) looked at the possibility that Jupiter might be a carbon-rich planet and examined element-by-element the fit to the Galileo probe data of two contrasting models: one in which the planetesimal building blocks of Jupiter derived from a disk with 
$\mathrm{C} / \mathrm{O}=1 / 2$ (roughly, the solar value), and the other in which $\mathrm{C} / \mathrm{O}=1$. To achieve the latter they assumed that the oxygen abundance in the disk had been depleted by a factor of two.. They then invoked planetesimal trapping in clathrate hydrates that provides a good fit to the elemental abundances in the Galileo probe data for the $\mathrm{C} / \mathrm{O}=$ solar case (Mousis et al. 2009, and reexamined the fit in the case for which $\mathrm{C} / \mathrm{O}=1$. Within the error bars of the probe data, there is little to distinguish the two bulk elemental abundances the one from the other. Both fit carbon, nitrogen, sulfur and argon equally well. Neither fits phosphorous, krypton or xenon, overestimating all of them. The solar C/O case is closer to $\mathrm{P}$ and $\mathrm{Xe}$ than the carbon-rich case, but both are well outside the probe error bars. Mousis et al. (2011) point out that the measured phosphorous abundance is likely a lower limit to the bulk abundance of phosphorous in Jupiter, because highly non-volatile phases may be carrying much of this element. Krypton and xenon cannot be so explained, but Mousis et al. (2009) had already invoked complexing of these noble gases with $\mathrm{H}_{3}^{+}$as a way of limiting their trapping in clathrate hydrates in the protoplanetary disk.

Regardless of the details, the real test is in the oxygen abundance, which must be seven times solar in Jupiter in the $\mathrm{C} / \mathrm{O}=$ solar case to account for the trapping of the volatiles, and roughly solar in the $\mathrm{C} / \mathrm{O}=1$ case. In both cases, the $\mathrm{O} / \mathrm{H}_{2}$ abundance in the Jovian envelope is larger than that in the protoplanetary disk overall because of the inefficiency of trapping of the volatiles in the ice. For direct condensation of all volatiles with no clathrate, the water enhancement is about 4 times solar in the $\mathrm{C} / \mathrm{O}=$ solar case and below solar in the $\mathrm{C} / \mathrm{O}=1$ case (remember that Mousis et al. (2011) label these cases by the $\mathrm{C} / \mathrm{O}$ values in the source material of the planetesimals in the original disk). The hypothesis of Lodders (2004), that the volatiles are actually trapped within organic solids, would lead to an even smaller water abundance in Jupiter. Thus, any determination of the deep water abundance in Jupiter yielding a value relative to hydrogen of solar or less would be a strong argument in favor of a carbon-rich Jupiter.

All of this begs the question of the source of the depletion. A wide range of oxidation states existed in the protoplanetary disk at different times and locations. For example,

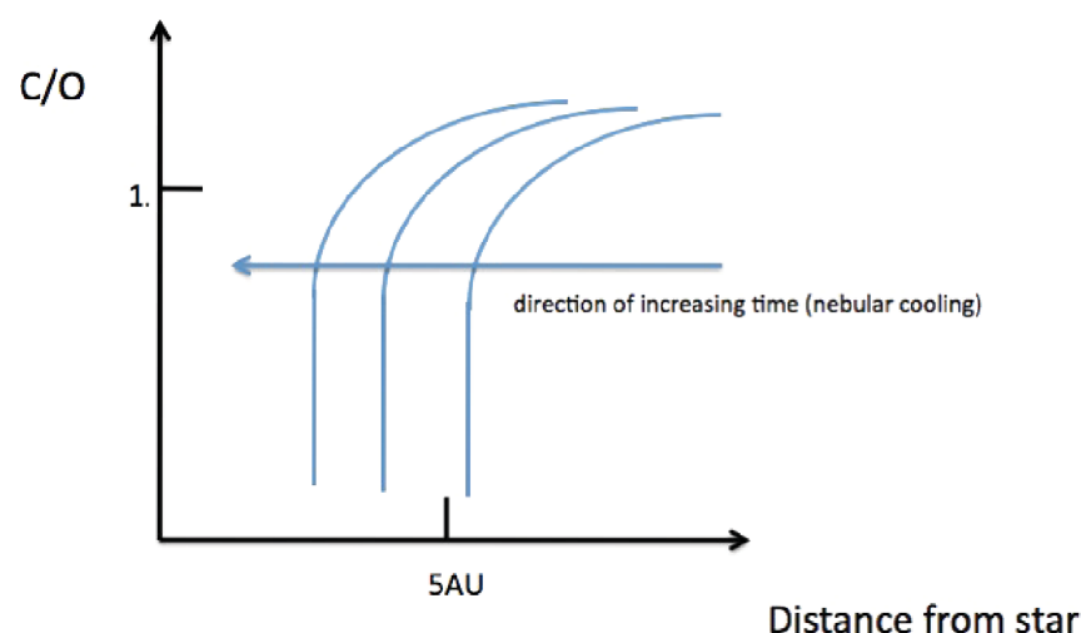

Figure 3. Qualitative behavior of the snowline- the radial position at which water ice condenses-in a protoplanetary disk, for varying elemental ratios of carbon to oxygen. Because silicates and metals sequester some of the oxygen at high temperatures, the amount of water drops precipitously and the snow line moves rapidly outward for $\mathrm{C} / \mathrm{O}$ values approaching, but still below, unity. 
the enstatite chondrites are thought to have come from parent bodies formed inward of all the other parent bodies, and their mineralogy suggests reducing conditions-consistent with a depleted water vapor abundance-in the region of the nebula where they formed (Krot et al. 2000). That region was almost certainly inward of the nebula snowline, where radial zones of enrichment and depletion of water vapor associated with the cold finger effect of condensation and inward radial drift of the icy particles existed (Ciesla and Charnley 2006. Recent models of the early evolution of Jupiter and Saturn hypothesize that these giant planets moved inward significantly during the late stages of their formation, reaching 1.5 AU in the case of Jupiter (Walsh et al. 2011), almost certainly inward of the snowline (Fig. 4). If planetesimals in this region accreted volatiles on refractory organic and silicate surfaces, as in the Lodders (2004) idea, which were then incorporated into Jupiter, the latter would appear carbon-rich and oxygen-depleted. However, temperatures in that region may not have been low enough to provide sufficient amounts of the more volatile phases. Further, this seems inconsistent with the nitrogen isotopic measurements for Jupiter, which suggest different molecular carriers for nitrogen in Jupiter versus the terrestrial planet region.

A second possibility is that Jupiter's migration scattered these water poor planetesimals to the colder outer solar system, where they trapped noble gases and carbon-and nitrogen-bearing species at lower temperature-but water ice, already frozen out, was not available. Again, these models are of interest only should it turn out that the oxygen depletion measured by Galileo reflects a bulk oxygen depletion, rather than being atmospheric. Essential is the measurement of this quantity, in Jupiter and if possible, in Saturn.

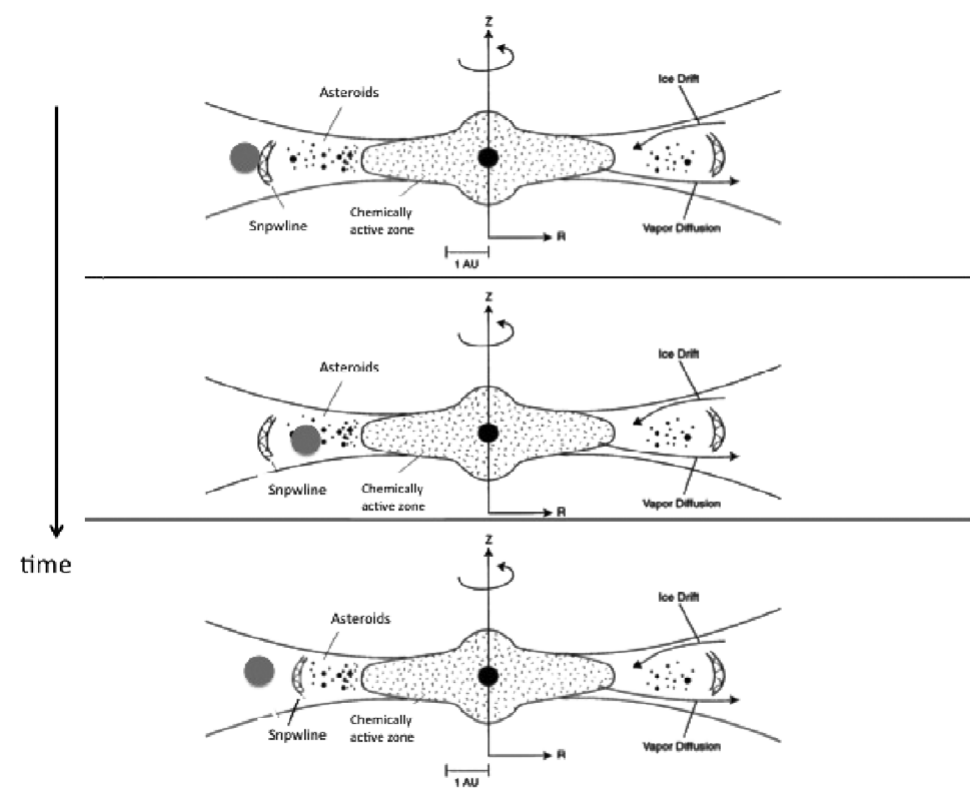

Figure 4. Schematic of the movement of Jupiter and the nebular snow line. In the top panel, Jupiter represented by the grey disk lies beyond the snowline, represented by the crescent. In the middle panel, later, Jupiter has moved inward of the snowline thanks to the influence of Saturn (Walsh et al. 2011). In the bottom panel, Jupiter has moved out again, while the snowline is moving inward at much slower pace (longer timescale). Base figure from Cyr et al. 1998 


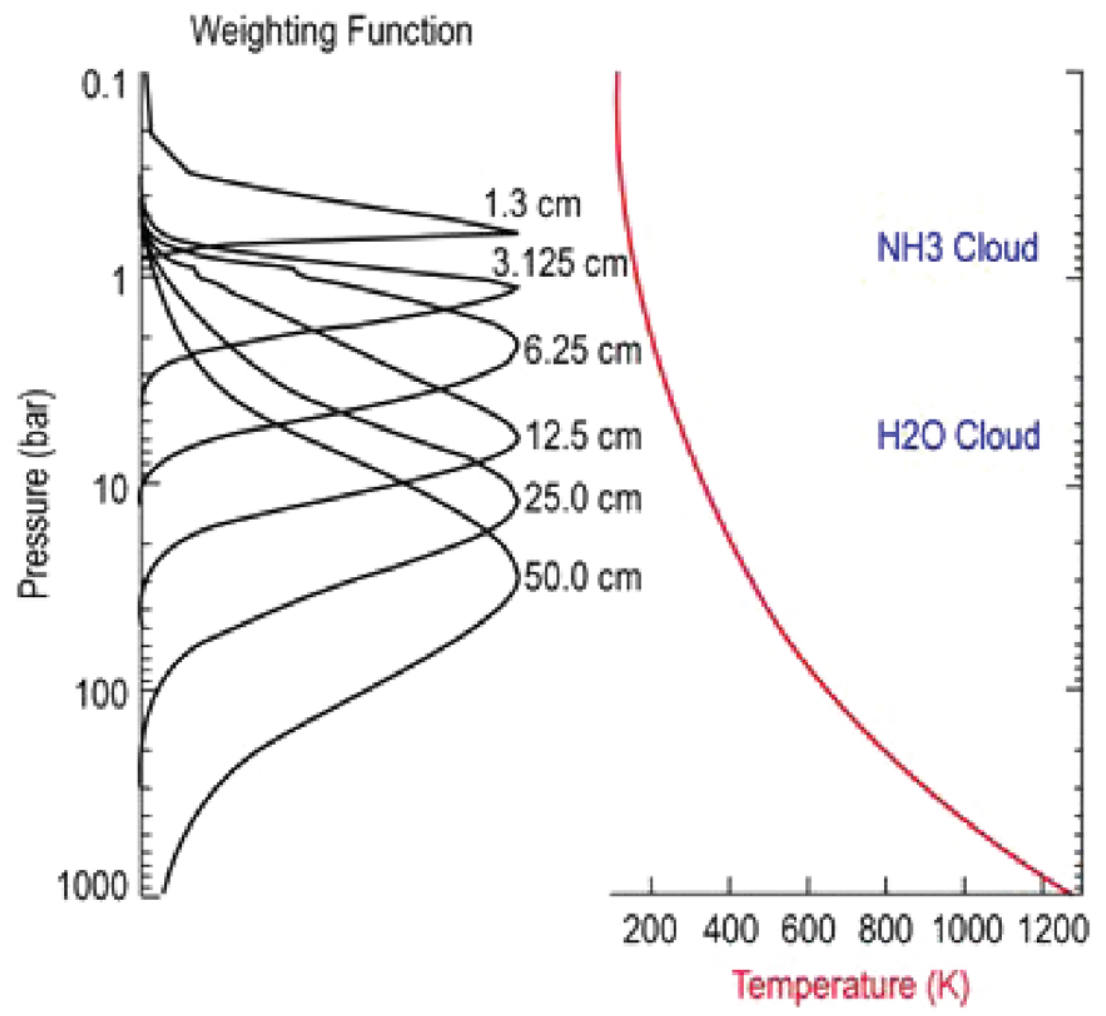

Figure 5. Weighting functions for the microwave radiometer on Juno, showing that the instrument is sensitive to water abundances at tens of bars bar pressure levels on Jupiter. Notional cloud decks are shown on the right based on the drawn temperature profile and the vapor pressures of the cloud formers. From Janssen et al. (2005).

\section{The future: measuring oxygen in Jupiter and noble gases in Saturn}

August 5, 2011 marked the launch of NASA's Juno mission to Jupiter, carrying eight instruments including two from the Italian Space Agency (ASI). Among the instrument suite is a microwave radiometer (MWR) built at NASA's Jet Propulsion Laboratory (Janssen et al., 2005) with the capability of measuring the abundance of water down to many tens of bars below the Galileo limit (Fig. 5). Complementary to the MWR is a near-infrared spectrometer JIRAM (Jovian Infrared Auroral Mapper), built by ASI, that will obtain the water abundance in the meteorological layer (Adriani et al. 2008), thereby providing a cross-check for the water abundance measured at the shortest wavelengths by the MWR. The two instruments together will be able to provide a definitive answer for whether the water abundance is below or above solar, and in the latter case, by how much. Measurements will begin when Juno enters Jupiter orbit in summer 2016.

Further in the future is the possibility of sending a probe into the Saturn atmosphere akin to Galileo. Such a mission would complement the detailed remote sensing being done by the Cassini Saturn Orbiter, which beginning in 2016 will make very close flybys of Saturn similar to what Juno will do at Jupiter, to map the gravitational and magnetic fields, as well as examine the atmospheric composition at close range in the infrared. Unfortunately, a microwave radiometer akin to that on Juno is not present on 


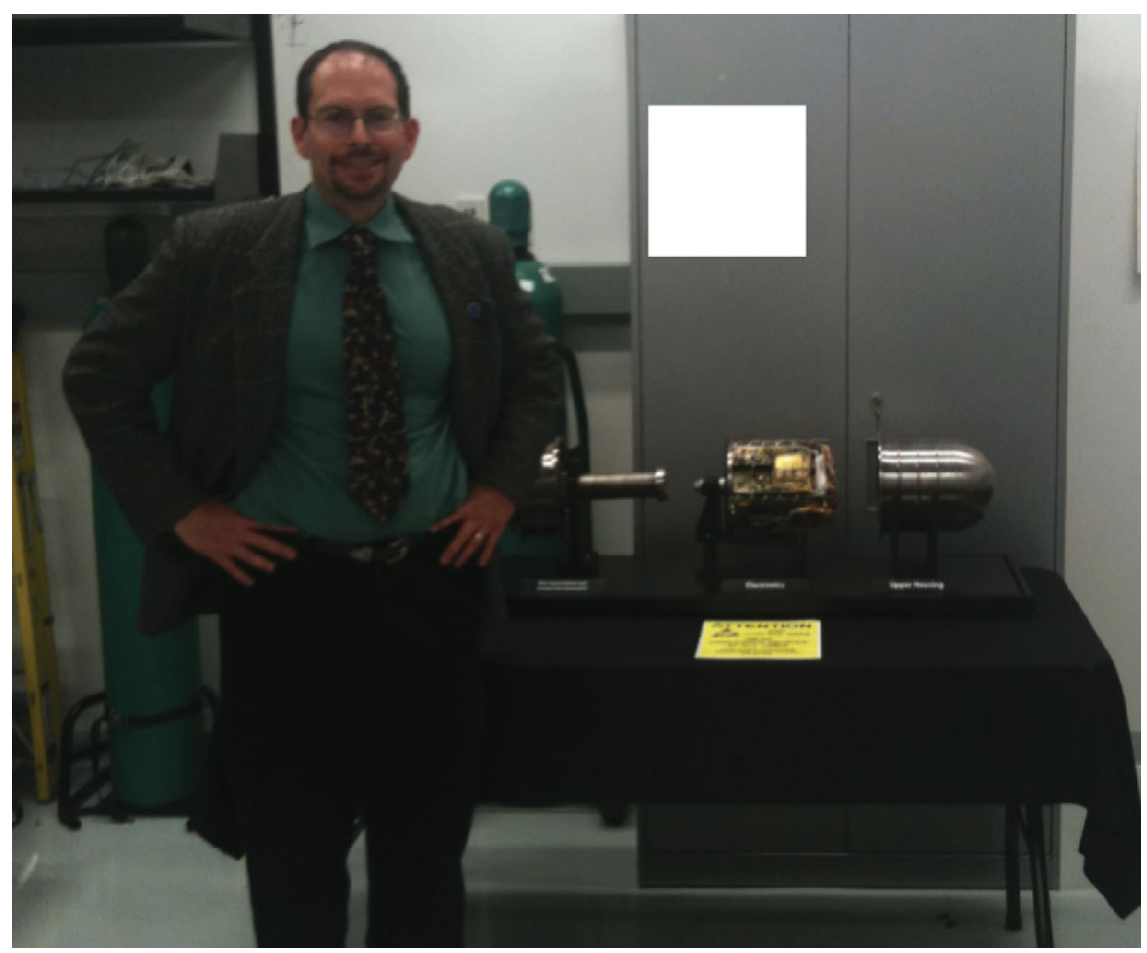

Figure 6. The author in front of a duplicate of the Galileo Probe mass spectrometer (on table) built by Hasso Niemann of the NASA Goddard Spaceflight Center. The detector is on the left; electronics in the center, and the upper housing on the right. Photo by M. Amato.

the Cassini Orbiter. Current strategic plans for the US planetary program (Space Studies Board 2011) limit Saturn Probe missions to the medium "New Frontiers" class, which means that a very deep probe (100 bars), will not be practical. Nonetheless, even flying a current version of the venerable Galileo Neutral Mass Spectrometer (Fig. 6) will provide a wealth of data to compare with that from Jupiter, and the possibility of deep probing of the Saturn atmosphere by microwave radiometry from a carrier, to complete the compositional diagnosis by obtaining the oxygen abundance, remains under study.

The fascinating possibility that Jupiter and Saturn might have distinct oxygen abundances because they sampled at different times and to differing extents regions of the nebula heterogeneous in oxygen (i.e., water) abundance is testable if a Saturn probe can measure the bulk oxygen abundance. In particular, should Saturn have a significantly higher $\mathrm{C} / \mathrm{O}$ abundance than Jupiter (even if both were above solar), it would support the idea that the region inward of the nebular snowline was significantly depleted in water, giving us a unique insight into nebular condensation and transport processes. Complementary to this information are the "hot Jupiters" like WASP12-b which are easier targets for measuring $\mathrm{C} / \mathrm{O}$ ratios, because their elevated temperature profiles allow both water and carbon-bearing species to be measured without interference from condensation processes (although cloud-forming species more refractory than water ice can reduce spectral contrast for some temperature ranges). 


\section{Implications for the origin of water on terrestrial planets}

In this chapter I have pointed out that we know less about the bulk elemental abundance of the solar system's largest reservoir of planetary material than one might have otherwise assumed. Another area of deep ignorance is the origin of Earth's water, which recently was succinctly summarized (Cowen 2011). Dynamical simulations argue against a cometary origin for Earth's water (Lunine 2011), as does the high cometary D/H ratio (see this conference)-but with the caveat of Brown et al. (2011). This leaves local sources such as may be derived from adsorption onto grains (de Leeuw et al. 2010), or the asteroid belt. However, while meteorite water contents suggest there is plenty of water in main belt carbonaceous chondrite parent bodies, matching the geochemical constraints is difficult (see the discussion and references in Lunine et al. 2011). Others have suggested the possibility that the main belt comets might be examples of bodies from which the water was derived (Hsieh and Jewitt, 2006; see also the discussion in Lunine et al. 2011). However, we know little about these bodies.

The notion that Jupiter and Saturn might have moved significantly in terms of radial distance in the final stages of their formation makes it more likely that icy bodies from beyond the snowline were introduced into the terrestrial planet region, making the acquisition of water on the Earth easier. However, we do not have any information on the $\mathrm{D} / \mathrm{H}$ values for water in this material, unless we think that these bodies are similar to comets, in which case $\mathrm{D} / \mathrm{H}$ is not consistent with the terrestrial value. And the water adsorption hypothesis invokes material for which we have no geochemical information at all. It is a daunting problem.

Ultimately, there may be a connection between the problem of the elemental abundances of our giant planets and the sources of water for the Earth. The material that Jupiter and Saturn swept up in the late stages of their formation should be related to that which they threw outwards, to make the comets, and inward to seed the terrestrial planets with water and organics. And so determining the full set of elemental abundances in Jupiter and Saturn is a part of the process of testing hypotheses for how the Earth acquired its water. Whether there is enough information to make headway-and whether studies of the elemental abundances in extrasolar giant planets relative to their parent stars will reveal important systematics-will not be known for some time to come.

\section{Acknowledgement}

The talk was presented while the author was a Visiting Professor at the University of Rome, Tor Vergata, Rome Italy. Some of the work was financed within the scope of the program "Incentivazione alla mobilita' di studiosi straineri e italiani residenti all'estero." This work was also supported by NASA through the Juno mission to Jupiter.

\section{References}

Adriani, A., Coradini, A., Filacchione, G., Lunine, J. I., Bini, A., Pasqui, C., Colamai, L., Colosimo, F., Dinelli, B. M., Grassi, D., Magni, G., Moriconi, M. L., \& Orosei, R. 2008 Astrobiology, 8, 613

Asplund, M., Grevesse, N., Jacques Sauval, A., \& Scott, P. 2009 ARAA, 47, 481.

Brown, R. H. B., Lauretta, D. S., Schmidt, B., \& Moores, J. 2011, PSS, in press.

Ciesla, F. J. \& Carnley, S. B. 2006, in: D. S. Lauretta and H. Y.McSween (eds.), Meteorites and the Early Solar System II (Tucson: University of Arizona Press), p. 209.

Cowen, R. 2011 Science News January 15 , 2011, 26

Cyr, K. E., Sears, W. D., \& Lunine, J. I. 1998 Icarus, 135, 537

de Leeuw, N. H., Catlow, R. A., King, H. E., Putnis, A., Muralidharan, K., Deymier, P., Stimpfl, M., \& Drake, M. J. 2010 Chem. Comm., 46, 8923. 
dePater, I., Dunn, D., Romani, P., \& Zahnle, K. 2001, Icarus, 149, 66

Fouchet, T., Moses, J. I., \& Conrath, B. J. 2009, in: M. K. Dougherty, L. W. Esposito \& S. M. Krimigis (eds.), Saturn from Cassini-Huygens (Dordrecht: Springer), p. 83

Grevesse, N., Asplund, M., \& Sauval, A. J. 2005, in G. Alecian, O. Richard, S. Vauclair (eds.), Element stratification of stars, 40 years of atomic diffusion (Les Ulis: EDP Sciences), p. 21

Hanel, R. A., Conrath, B. J., Kunde, V. G., Pearl, J. C., \& Pirraglia, J. A. 1983 Icarus, 53, 262

Hersant, F., Gautier, D., \& Lunine, J. I. 2004, Planetary and Space Science, 52, 623

Hsieh, H. \& Jewitt, D. C. 2006, Science, 312, 561

Hubbard, W. B., Dougherty, M. K., Gautier, D., \& Jacobson, R. 2009, in: M. K. Dougherty, L. W. Esposito \& S. M. Krimigis (eds.), Saturn from Cassini-Huygens (Dordrecht: Springer), p. 75

Janssen, M. A., Hofstader, M. D., Gulkis, S., Ingersoll, A. P., Allison, M. A., Bolton, S. J., Levin, S. M., \& Kamp, L. W. 2005, Icarus, 173, 447

Krot, A. N., Fegley, B., Jr., Lodders, K., \& Palme, H. 2000, in: V. Mannings, A. P. Boss \& S. S. Ressell (eds.), Protostars and Planets IV (Tucson: University of Arizona Press), p. 1019.

Lodders, K. 2004, ApJ, 611, 587

Lodders, K. \& Fegley, B. 1998, Meteorit. Planet. Sci., 33, 871

Lunine, J. I., O'Brien, D. P., Raymond, S. N., Morbidelli, A., Quinn, T. \& Graps. A. L. 2011 Advanced Science Letters4, 325.

Lunine, J. \& Hunten, D. M. 1987, Icarus, 69, 566

Madhusudhan, N., Harrington, J., Stevenson, K., Nymeyer, S., Campo, C., Wheatley, P., Deming, D., Blecic, J., Hardy, R., Lust, N., Anderson, D., Collier-Cameron, A., Britt, C., Bowman, W., Hebb, L., Hellier, C., Maxted, P., Pollacco, D., \& West, R. 2011, Nature, 64, 469

Madhusudhan, N., Mousis, O., Johnson, T. V., \& Lunine, J. I.2011, ApJ in press

Mahaffy, P. R., Niemann, H. B., Alpert, A., Atreya, S. K., Demick, J., Donahue, T. M., Harpold, D. N., \& Owen, T. C. 2000, J. Geophys. Res., 105, 15,061.

Marty, B., Guillot, T., Coustenis, A., \& 55 others 2008 Experimental Astronomy DOI $10.1007 /$ s10686-008-9094-9

Mousis, O., Madhusudhan, N., Lunine, J. I., \& Johnson, T. V. 2011, ApJ, submitted.

Mousis, O., Marboeuf, U., Lunine, J. I., Alibert, Y., Fletcher, L. N., Orton, G. S., Pauzat, F., and Ellinger, Y. 2009, ApJ, 696, 1348.

Niemann, H. B., Atreya, S. K., Carignan, G. R., Donahue, T. M., Haberman, J. A., Harpold, D. N., Hartle, R. E., Hunten, D. M., Kasprzak, W. T., Mahaffy, P. R., Owen, T. C., \& Way, S. H. 1998, J. Geophys. Res., 103, 22,831

Owen, T., Mahaffy, P. R., Niemann, H. B., Atreya, S., Donahue, T., Bar-Nun, A., \& de Pater, I. 1999, Nature, 402, 269

Owen, T. \& Encrenaz, T. 2003, Space Science Rev., 106, 121

Proffitt, C. R. 1994, ApJ, 425, 849

Showman, A. P. \& Dowling, T. E. 2000, Science, 289, 1737

Space Studies Board 2011 Vision and Voyages for Planetary Science in the Decade 2013-2022 (Washington: National Academies Press)

Stevenson, D. J. \& Salpeter, E. E.1977 ApJ Suppl, 35, 221

Wong, Von Zahn, U., Hunten, D. M., \& Lehmacher, G. 1998 JGR 103, 22815

Walsh, K. J., Morbidelli, A., Raymond, S. N., O'Brien, D. P., \& Mandell, A. M. 2011, Nature, 475,206

Wilson, H. F. \& Militzer, B. 2010, Physical Review Letters, 104, 147

Wong, M. H., Lunine, J. I., Atreya, S. K., Johnson, T., Mahaffy, P. R., Owen, T. C., \& Encrenaz, T. 2008 Reviews in Mineralogy $\&$ Geochemistry. 68, 219.

\section{Discussion}

VAN Dishoeck: Can you elaborate on the similarities and differences between Main Belt comets and normal comets, in terms of composition, especially water/organics ratio?

LuninE: To my knowledge we have no information on the composition of the Main Belt comets, certainly not the level of detail needed for the ratio of water to organics. 
IRVINE: Do we know enough about cometary D/H to rule out traditional comets as the source of Earth's water?

LuninE: Yes, one can rule out comets, if one assumes that the $\mathrm{D} / \mathrm{H}$ value observed in the cometary comae are representative of the value in the bulk nucleus. Although it seems to be a reasonable assumption, there are several papers that argue $\mathrm{D} / \mathrm{H}$ values may be significantly altered by vaporization processes ( for example, Brown et al. 2011). 